\title{
Propuesta del Modelo de Cadena de Valor de la Cebolla Junca de Aquitania, Boyacá
}

\author{
Value Chain Mode of Cebolla \\ Junca of the Aquitania, Boyacá
}

\section{Resumen}

Artículo de investigación.

Fecha de recepción: 26/02/2020

Fecha de devolución: 18/06/2020

Fecha de aceptación: 25/06/2020

Fecha de publicación: 03/07/2020

\section{Jenny Mairena Herrera Rodríguez \\ Universidad de Boyacá. \\ Sogamoso (Colombia) \\ jenmaiherrera@uniboyaca.edu.co}

\section{Wilmar Nicolas Chacón Casallas Universidad de Boyacá. \\ Sogamoso (Colombia) \\ wnchacon@uniboyaca.edu.co}

\section{Julián Andrés Aragón García \\ Universidad de Boyacá. \\ Sogamoso (Colombia) \\ jularagon@uniboyaca.edu.co}

Para citar este artículo:

Herrera Rodríguez, J., Chacón Casallas, W. \& Aragón García, J. (2020). Propuesta del Modelo de Cadena de Valor de la Cebolla Junca de Aquitania, Boyacá Económicas CUC, 41(2), 137-148. DOI: https://doi. org/10.17981/econcuc.41.2.2020.Org.8
En un mercado globalizado y saturado de bienes y servicios, en el cual todos son commodities, la cadena de valor permite añadir factores diferenciadores a lo largo de su producción que da como resultado un valor agregado, el cual permite la preferencia en la decisión de compra y la competitividad en los mercados globalizados. Este trabajo corresponde a los resultados parciales de un proyecto de investigación. El presente artículo busca proponer un modelo para la cadena de valor de la cebolla Junca de Aquitania, identificando los actores que intervienen directa o indirectamente desde la siembra hasta su comercialización al por mayor. La metodología utilizada es mixta, la recolección de datos se realiza por medio de observación, entrevista, análisis documental, el instrumento a utilizar es un cuestionario diseñado por los autores. Se concluye que la cadena de valor es una de las herramientas más eficaces que existen puesto que, una vez comprendida facilita los procesos de innovación, mejora continua o toma de decisiones organizacionales.

Palabras clave: Cadena de valor; ventaja competitiva; Aquitania; Boyacá; Cebolla Junca

\section{Abstract}

In a globalized and saturated market of goods and services, in which all are commodities, the value chain allows differentiating factors to be added throughout its production, which results in added value, which allows preference in the purchase decision and competitiveness in globalized markets. This work corresponds to the partial results of a research project. This article seeks to propose a model for the Junca Aquitaine onion value chain, identifying the actors that intervene directly or indirectly from planting to wholesale marketing. The methodology used is mixed, data collection is done through observation, interview, documentary analysis, the instrument to use is a questionnaire designed by the authors. It is concluded that the value chain is one of the most effective tools that exist since, once understood, it facilitates the processes of innovation, continuous improvement or organizational decision-making.

Keywords: Value chain; competitive advantage; Aquitaine; Boyacá; Onion Junca 


\section{INTRODUCCIÓN}

El concepto de cebolla proviene del fondo léxico mediterráneo del latín cepulla. "La cebolla es el bulbo subterráneo y comestible que crece en la planta del mismo nombre" (López, 2017, p. 2).

Actualmente existe una amplia gama de variedades, que pueden clasificarse en función del color del bulbo, forma, tamaño, usos, origen y precocidad. Tiene muchos usos culinarios, pudiendo usarse de distintas maneras, ya sea cruda o cocinada. Además, se le conocen distintas propiedades medicinales (Interempresas Media, 2019, p. 1).

La cebolla es un alimento fundamental en los platos de muchas familias colombianas, ofreciendo un sabor potente y nutritivo. Fue introducida a Colombia por los españoles. Y en la actualidad, según la Encuesta Nacional Agropecuaria, ENA (Departamento Administrativo Nacional de Estadística, DANE, 2015) "durante el año 2013 en Colombia se cosecharon 14.533 hectáreas de cebolla de rama o cebolla Junca con una producción de 289.975 toneladas y rendimiento de 39,9 toneladas por hectárea al año" (p. 2).

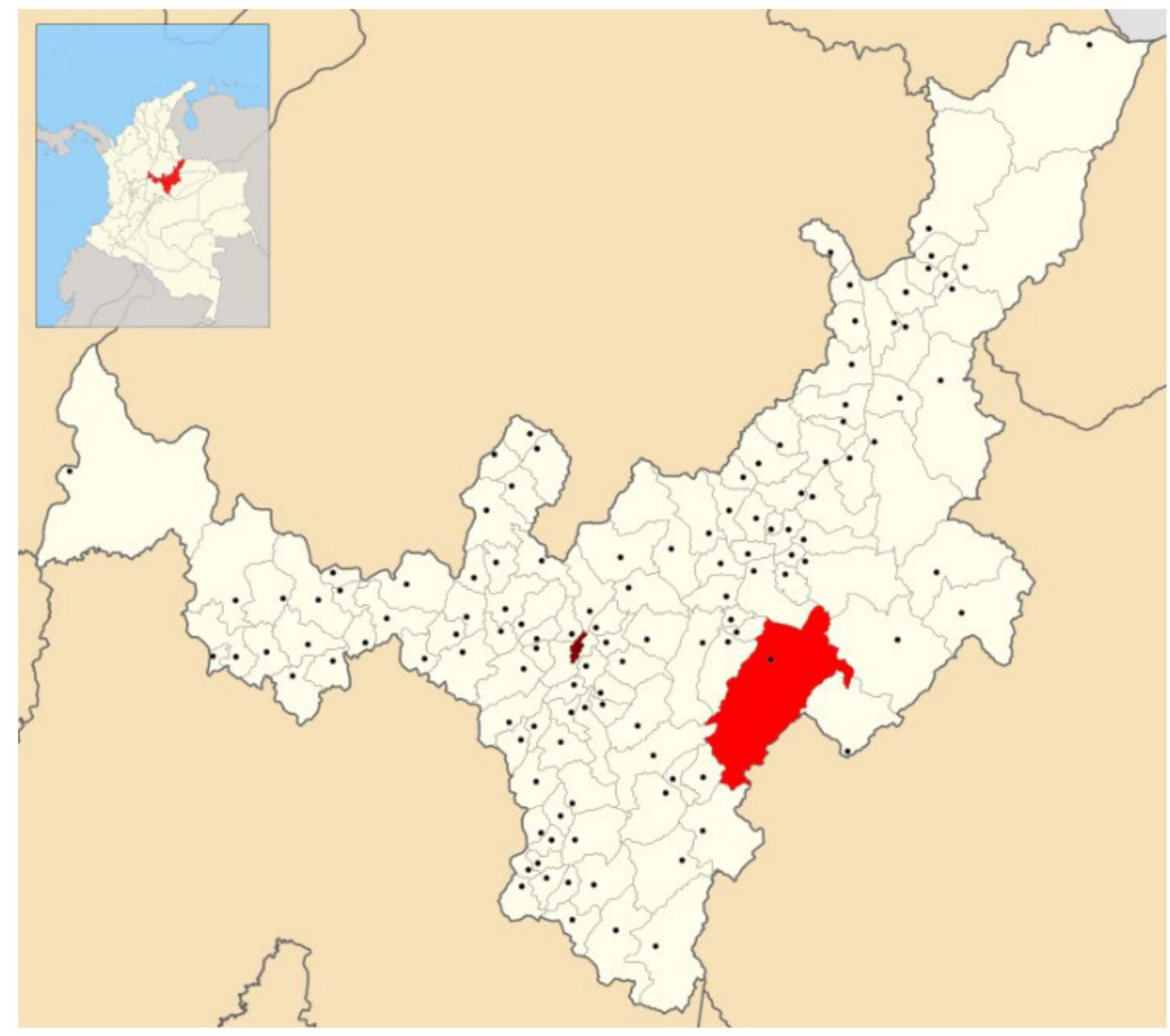

Figura 1. Mapa del Municipio de Aquitania, Boyacá (Colombia). Fuente: Milenioscuro, 2012 [Trabajo propio, cc by 3.0]. https://es.m.wikipedia.org/wiki/Archivo:Colombia_-_Boyaca_-_Aquitania.svg 
La zona objeto de estudio es Aquitania- Boyacá, ubicada a 300 Kilómetros de la ciudad de Bogotá la capital de Colombia (Figura 1). Posee una ubicación estratégica sobre estribaciones de la cordillera Oriental, su área limita con 10 municipios de Boyacá (Alcaldía Municipal de Aquitania, 2018), los cuales los describe la alcaldía de Aquitania de esta manera:

- El norte: Sogamoso, Cuitiva y Mongua.

- En el sur: Zetaquira, San Eduardo y Paez.

- En el oriente: Labranzagrande, Pajarito, Recetor y Chámeza.

- En el occidente: Cuitiva, Tota, Zetaquira y San Eduardo.

- Extensión total: 943 kilómetros cuadrados $\mathrm{Km}^{2}$.

- Extensión área urbana: 0.52 kilómetros cuadrados $\mathrm{Km}^{2}$.

- Extensión área rural: 827.48 kilómetros cuadrados $\mathrm{Km}^{2}$.

- Altitud de la cabecera municipal (metros sobre el nivel del mar): 3.030 metros sobre nivel del mar.

- Temperatura media: $10^{\circ} \mathrm{C}$.

Boyacá se convierte en el mayor productor de cebolla Junca de Colombia, siendo Aquitania su principal productor y fuente económica en la actualidad, en el cual inició con las primeras siembras a comienzos de los años sesenta a cultivar la cebolla Junca en las orillas de la Laguna de Tota.

En Colombia persiste la discusión del bajo crecimiento del primer trimestre del año 2019. Según el DANE "la economía colombiana creció en $2,8 \%$ durante el primer trimestre del año" (Medina, 2019, p. 1), datos que muestran que el país estuvo por debajo de las expectativas. La máxima autoridad de estadística del país reveló un desempleo nacional de $12.8 \%$ en enero que, en cierto modo, no llegaba a ser tan alto desde el 2011, cuando fue de 13,6\%. Esto es debido a la pérdida de 140 mil empleos en el agro según el DANE, hecho que genera interrogantes que merecen ser investigados.

Además, según el Instituto Geológico Agustín Codazzi (IGAC) el territorio colombiano cuenta con 114 millones de hectáreas aproximadamente, 54\% en manos de propietarios privados. En el índice Gini Colombia muestra un 89,7 de desigualdad, siendo cien (100) la plena desigualdad (El Tiempo, 2016). Más aún cuando el informe de IGAC da cuenta de que los departamentos con mayor cantidad de predios de un único propietario son Antioquia, Cundinamarca y Boyacá (El Tiempo, 2016). Que revelan el sufrimiento del pequeño campesino boyacense en generar desarrollo y una calidad de vida aceptable para su sostenimiento. Es por esto, que el uso de la tierra ha venido perdiendo dinamismo en su crecimiento y muchos de sus empleos son informales y de baja calidad. Otorgando cifras que hagan difícil el desarrollo en un departamento caracterizado por su agro, como lo es Boyacá. Por ende, la tierra para uso agrícola se está desperdiciando y no está siendo utilizada eficientemente para generar productividad económica y empleo en la población rural. 
Es necesario promover la exportación de productos y servicios, que contribuyan al desarrollo económico de Boyacá y que lleven al pequeño campesino a ser competitivo. En el municipio de Aquitania-Boyacá. La cebolla Junca es la principal fuente de empleo (Bayer Crop Science, 2014), cuentan con una tierra rica en fuentes hídricas e innumerables recursos naturales que podrían impulsar la economía en esa región.

Para Giovanni Contreras, un agricultor mayorista con una larga tradición de familia en el negocio, "no existe una mejor cebolla Junca en Colombia que la producida en Aquitania". La considera un producto único que, a futuro, debería recibir denominación de origen. "Su calidad marca la diferencia gracias a que es cultivada a una gran altura, pero sin neblina por efecto del Lago de Tota. Tiene un sabor picante y agradable, sus tallos suelen ser más largos que los de otras y posee un valor nutricional altísimo. La gente siempre prefiere la cebolla de Aquitania" (Bayer Crop Science, 2014, párr. 6).

Para analizar las diferentes actividades que intervienen e proceso, se ha de tener en cuenta una de las características principales de la agricultura y que a la vez también es importante el cultivo de la cebolla de rama:

[...] las unidades de producción son heterogéneas en capital, tecnología, administración, capacidad e información; ello contribuye a que la oferta, por el lado de los productores primarios sea altamente competitiva con ausencia de coordinación y con escasa o nula planificación para ajustarse a las necesidades de la demanda (Bejarano, 1998, p. 93).

Para que el sector de la cebolla larga pueda establecerse en el mercado de una manera más estable, primero necesitan de una descripción clara que permita comprender la cadena de valor de la cebolla Junca del municipio de Aquitania, como también, las características especiales que lo diferencien ante otras cebollas del país. Luego se debe determinar factores que inciden en sus condiciones especiales, las cuales pueden ser factores humanos y naturales que difieran en el producto. Según Forero (2017), se hace necesario reconocer los actores y procesos de la cadena de valor para generar competitividad, reducción de costos y procesos que se identifiquen como necesarios para una eficiencia en el mercado. Aquitania necesita determinar las ventajas competitivas y actividades estratégicas más relevantes que caracterizan que el producto sea especial.

Siendo este municipio el mayor productor de cebolla, se analizan varias dificultades en cuanto a su competitividad en el mercado, pues como lo afirma (Bayer Crop Science, 2014), la Cebolla Junca es la principal fuente de empleo y Aquitania cuenta con una tierra rica en fuentes hídricas e innumerables recursos naturales que podrían impulsar aún más la economía en esa región. La revisión de literatura no arrojó trabajos científicos que describieron las diferentes actividades inherentes a esta cadena de valor, pero si algunos artículos sobre trabajos relacionados a la cebolla de Aquitania. Es por esto que se buscó desarrollar una investigación cuyo objetivo apunta a la propuesta de cadena de valor de este producto buscando una ventaja diferenciadora, pero para llegar a ser competitivos y este artículo busca proporcionar una base fundamental para el desarrollo del negocio. 


\section{DesarRollo}

\section{Investigaciones acerca de modelos de cadena valor en la agricultura}

Argueta, Cardona, Albán y Moreno (2014) estudiaron la inserción de lulo deshidrato colombiano en la cadena de valor del mercado peruano, con el fin, identificar oportunidades de negocio que puedan tener el sector agrícola y propagar el sector de alimentos reconocido en Perú. La intención de este estudio es resaltar el Lulo como opción en el mercado peruano, buscando fortalecer las relaciones comerciales para beneficio mutuo.

El modelo aplicado es una investigación exploratoria realizada en Lima (Perú), instaurado por la Uniempresarial (entidad donde se realiza acercamiento de empresas y universidades), con el fin de establecer la posibilidad de introducción del Lulo deshidrato en el mercado peruano (Jiménez y Molano, 2019). Según lo recopilado en este estudio, el ambiente es apto para la comercialización de alimentos agrícolas gracias a que los mercados globales están exigiendo variedad e innovación en los productos alimenticios por parte de los consumidores (Jiménez y Molano, 2019) Para la introducción de otros productos como loes la cebolla Junca, debemos tener en cuenta su cadena de valor y sus ventajas competitivas para su posterior comparación con otras industrias como lo es la peruana, donde la demanda de alimentos ha crecido con su marca Perú.

Así, las investigaciones de cadena de valor en Colombia, en los últimos 5 años, proveen una crisis en falta de desarrollo en la parte agraria en Colombia, descubriendo incapacidades en el momento de competir con otros países que ya reconocen detalladamente su cadena de valor para su posterior crecimiento continuo. El desarrollo empírico que demuestra el país ha dado como resultado una eficiencia a la hora de competir en el mercado global.

Para entender el tema principal de la investigación, se hace necesario mencionar a Porter, quien definió la cadena de valor como la acumulación de valor dentro de una serie de actividades, el cual tiene como finalidad un servicio o producto (Porter, 1985). El autor expresa las actividades internas a realizar que desempeña una compañía u organización para diseñar, producir, comercializar, entregar y respaldar un producto o servicio. Por otro lado, "la herramienta de análisis que permite ver hacia adentro de la empresa, en búsqueda de una fuente de ventaja en cada una de las actividades que se realizan" (Olmedo y Plazaola, p. 1).

Como lo menciona (Alonso, 2008), "la cadena de valor como una poderosa herramienta de análisis para la planeación estratégica de los negocios, básicamente, facilitando la identificación de ventajas competitivas en el seno de la organización” (p. 86). Siendo que toda organización es posible de analizar para conocer la contribución de valor que genera desde las actividades principales o las que surge en la relación de estas.

Autores como (Nuñez y Possetto, 2009) aseguran que una cadena es:

Una serie de eslabones que centran sus actividades en la elaboración de un producto. Cada eslabón participa un grupo de actores económicos que realizan actividades semejantes, con similares procesos de generación de valor, derechos propietarios sobre un producto o servicio en un estado de valor definido y que transfieren este producto a los mismos clientes al mismo tiempo que reciben insumos de los proveedores (p. 87). 
Por otro lado, Francés (2006) expresa que "la cadena de valor proporciona un esquema coherente para diagnosticar la posición de la empresa respecto a sus competidores, además de un marco conceptual, para definir acciones tendientes a desarrollar una ventaja competitiva sostenible" (p. 15). Es decir, permite establecer la posición interna y/o externa en que se encuentra una organización.

\section{Cadena de valor como ventaja competitiva}

Según De la Fuente y Muñoz (2003), el instrumento analítico introducido por Porter utilizado para determinar las fuentes de ventaja competitiva es la cadena de valor. Este instrumento permite un despliegue de su empresa en "las actividades estratégicas relevantes para comprender el comportamiento de los costos y las fuentes de diferenciación existentes y potenciales" (De la Fuente y Muñoz, 2003, p. 5). Es decir, que, si las empresas desean generar una ventaja diferenciadora, deben conocer y entender cada uno de los procesos y las tareas que conforman a estos.

De la Fuente y Muñoz (2003) aseguran que la cadena de valor no es un elemento aislado, sino que está inserto en un sistema de valor donde se encuentra la cadena de valor de los diferentes actores que intervienen en el proceso de una organización, como lo son los proveedores, distribuidores, clientes, entre otros. En la cadena de valor en una organización, se identifican las principales actividades que crean valor para los clientes, también para las actividades de apoyo afines. La cadena de valor igualmente identifica los costos en lo que incide una organización durante las actividades que conforman y que desde luego se llevan a cabo "en el proceso productivo, lo que significa un elemento o factor imprescindible para fijar la estructura de costos de una organización" (De la Fuente y Muñoz, 2003, p. 6).

Desde un punto estratégico de Quintero y Sanchez (2006) la cadena de valor de una empresa es la "forma en la cual se desempeña cada una de las actividades refleja la evolución de su propio negocio" (p. 377). Debe comprenderse que la cadena de valor de empresas contrincantes sea diferente a las verticales, por ende se dificulta realizar una evaluación de los costos relativos en las empresas rivales (Quintero y Sánchez, 2006, p. 378).

$\mathrm{Al}$ agregar valor, las empresas deben prestar atención a sus planes no solo estratégicos sino también en la parte de mercadeo. Es decir, para que la organización sea más competitiva debe ser sólidas y organizadas en su interior, pero al mismo tiempo conseguir la mayor cantidad de información de los clientes. De esta manera podrá efectuar los cambios internos necesarios y/o solicitados para lograr dar respuesta a las exigencias de la demanda en el mercado en donde se busca valor, crear y entregar valor (Severich, 2012).

Severich (2012) asegura que la cadena de valor no solo debe ejecutarse de manera apta sino debe someterse a revisiones periódicas. Estas revisiones permitan potenciar el aprendizaje intrínseco de la organización y, de manera flexible, propician que podrían constituir ventajas competitivas, las cuales deben ser difíciles de copiar, ya que surgen del trabajo natural de la empresa y de su proceso único. Por lo 
tanto, el aprendizaje organizativo, se da en cada una de las etapas o eslabones que se comprenden en la cadena de valor en una empresa. Por este motivo, cada una de las actividades o tareas deben ser retroalimentadas con los conocimientos que desencadenan los mismos procesos precedentes.

Para lograr verdaderos cambios en la organización a través de la cadena de valor esta debe ser supervisada con frecuencia ya que los cambios que se realicen deben convertirse en lo que se denomina ventaja competitiva, por lo cual esta nueva ventaja competitiva debe ser lo más difícil de copiar para nuestros competidores que ya que cuesta dinero, tiempo y todos los recursos que esto pueda requerir.

\section{Metodología}

Con una investigación mixta se desarrolla un estudio exploratorio y descriptivo, para reconocer la situación actual de la cebolla Junca del municipio de AquitaniaBoyacá en los procesos que dan valor al producto que se ofrece. Con base en documentación teórica, identificando los factores ambientales, socioeconómicos y privados que inciden en la cadena de valor y además lograr definir las actividades estratégicas que generan ventajas competitivas en la región, con mejoras económicas y ambientales para la cebolla de Aquitania.

Las técnicas y herramientas de recolección de información que permitieron reconocer las diferentes actividades, la interacción entre productores, comercializadores, clientes y demás actores son la observación. La fase que continúa en este proyecto es la aplicación de una encuesta, instrumento que permitirá reconocer más a fondo los procesos que se han venido desarrollando hasta el momento para que el producto llegue al consumidor final.

También se acude a fuentes secundarias, realizando una minuciosa revisión de literatura basada en dos aspectos, el primero reconocer la importancia de establecer la cadena de valor y lo segundo identificar investigaciones desarrolladas sobre este producto. La tabulación se hará a través del software SPSS, con el análisis de información se podrá plantear el Modelo de Cadena de Valor para posteriormente hacer un trabajo con la comunidad, generando estrategias de competitividad.

\section{Resultados}

Al realizar la cadena de valor esta tiene impactos positivos tanto para la organización como para las personas que interfieren directa e indirectamente dentro del proceso que se analiza con la cadena valor. Entre los beneficios que esta otorga se encuentra la ventaja competitiva, la cual permite obtener y crear un factor o variable única que diferencie la organización frente a los competidores. Por otro lado, la cadena de valor permite identificar oportunidades de mejora en cada uno de los eslabones o etapas que definamos dentro del proceso que se está llevando a cabo. Cada una de estas etapas es analizada por las actividades que se realizan allí, dando la oportunidad de realizar mejoras por cada una de las actividades, lo cual facilita la ejecución de mejorar continuamente en un corto plazo. 
TABLA 1.

Comparación entre las relaciones del negocio tradicional y la cadena de valor

\begin{tabular}{lll}
\hline \multicolumn{1}{c}{ Aspecto } & \multicolumn{1}{c}{ Enfoque Tradicional } & \multicolumn{1}{c}{ Cadena de Valor } \\
\hline Información compartida & Escasa o ninguna & Amplia \\
Objetivo primario & Costo/precio & Valor/Cantidad \\
Orientación & Commodities & Producto diferenciado \\
Relación de poder & Desde la oferta & Desde la demanda \\
Estructura de la organización & Independiente & Interdependiente \\
Filosofía & Auto optimización & Optimización de la Cadena \\
\hline
\end{tabular}

Fuente: Iglesias (2002, p. 6).

Mediante el análisis comparativo entre el enfoque tradicional y su aplicación en una cadena de valor, se puede lograr identificar la deficiencia de este enfoque tradicional (Tabla 1). Está claro que se requiere un análisis basado en datos específicos para poder ratificar una estructura donde se otorgan herramientas que buscan entender y descifrar los factores que se pueden mejorar para crear un producto superior, centrándose en las necesidades que se ofrecen de una forma más práctica y visible, y que a partir de ahí se obtengan progresos como lo muestra la aplicación de la cadena de valor.

En la implementación de la cadena de valor, en comparación con el enfoque tradicional, se permite entender los costos y búsqueda de estrategias diferenciadoras que generan valor al producto final, ocasionado ventajas competitivas que son percibidas por los clientes, logrando así una mayor aceptación del bien o servicio. La cadena de valor se puede crear teniendo como base todos los procesos y actividades que se realizan, también se puede concebir con diferentes herramientas dentro del campo de la administración de empresas como lo son la matriz DOFA, benchmarking y el ciclo de vida del producto entre otros.

Una de las mayores necesidades por la cual debe realizarse la cadena de valor dentro de un ámbito de desarrollo e innovación es mencionada por las naciones unidas. Esta organización expresa que en los últimos años la concentración de la cadena de valor en el mundo, en cuanto a los productos básicos agrícolas, en donde se incluye la concentración horizontal y la integración vertical, ha provocado que un pequeño número de grandes empresas dominen la mayor parte del mercado, comenzando en la comercialización de los productos agrícolas, la etapa en el que se modifican dichos productos, hasta la venta al por menor. Tal es el caso del banano, ya que en las 2002 dos empresas dominaban el 50\% del comercio mundial de este producto, por otro lado, tres empresas monopolizaban tres cuartas partes del comercio mundial en los cereales.

Por lo general cuando uno de los objetivos principales que establece una organización es la producción en masa o producción a escala en la agricultura conlleva a efectuar una concentración de la cadena de valor en los productos que se desean 
producir. Aunque esta concentración de la cadena de valor también se puede realizar a varios factores como por ejemplo el deseo y necesidad de las organizaciones al realizar controles severos y estrictos por el hecho de garantizar la calidad que exigen sus demandantes por lo tanto esto crea una muralla para la entrada de nuevos actores y, a su vez, eliminan a pequeñas empresas que no tengan las facilidades o capacidades de competir en estos mercados monopolizados.

Otro factor que puede llegar a ser vital para la concentración de la cadena de valor es centralizar el valor, pero en uno de los eslabones o etapas, por ejemplo, en el procesamiento. Puede tener el mismo efecto en otros por ejemplo la fabricación, o la comercialización, esto puede influir de manera positiva o negativa en el poder de negociación de los eslabones más bajos de la cadena de valor llegando a desequilibrar, o no, la cadena de valor. Aquí radica el papel determinante de la planeación estratégicaen cuanto a la dirección que deseemos darle a la organización si es que se basa en la cadena de valor.

\section{DisCUSIÓN}

En el transcurso de la investigación se ha logrado evidenciar el impacto económico, ambiental y social que conlleva la producción de cebolla Junca, por cuanto cada vez reviste de más importancia el desarrollo de una investigación que apunta a fortalecer los procesos y actores que la intervienen. Un punto que vale la pena aclarar, es que en Boyacá la mayoría de las actividades de agricultura se realizan de manera artesanal, siendo la principal razón para no estar comercializando el producto en grandes esferas.

Las necesidades de los campesinos de ajustar sus prácticas productivas acorde a un modelo de globalización que demanda niveles de calidad, cumplimiento de normas, estandarización, que limita ampliar el mercado o darle valor.

Boyacá, específicamente Aquitania, cuenta con la laguna más grande de Colombia, de donde existe una gran tradición agrícola que depende económicamente la población, sin embargo, la agricultura que se está realizando de forma artesanal, ha generado una gran presión de contaminación en la laguna, fenómeno constante en Colombia. Se estudió el área en el 2019, los resultados de la investigación arrojaron diversos factores que impactan la laguna entre ellos la cebolla, acorde al artículo citaremos de tal forma como fue explicado en el documento:

La cuenca del Lago de Tota ha sido modificada por diversas actividades humanas, como deforestación, piscicultura y el monocultivo de cebolla larga (Allium fistulosum) con un elevado uso de agroquímicos y gallinaza cruda, causando deterioro de las características ecológicas del sistema léntico de alta montaña más grande de Colombia (Barrera, Andrea, Espinosa, Janneth, Álvarez y Pablo, 2019, p. 11).

En el departamento no se evidencian proyectos que busquen estructurar la Cebolla Junca, por lo que se hace necesario proponer un modelo de cadena de valor de la cebolla Junca de Aquitania-Boyacá, que contribuya económica y ambientalmente en el descubrimiento de procesos ineficientes o artesanales que afecten el impacto que se genera en la laguna. 
Uno de los grandes debates de esta investigación de modelo de cadena de valor, es generar ese impacto positivo de producción de cebolla Junca, que sea amigable al medio ambiente y a la sostenibilidad de la región, con el fin de otorgar una descripción que permita comprender los factores que promuevan la productividad eficiente y sostenible ambientalmente.

\section{Conclusiones}

Al hablar de todas las herramientas que existen para lograr cambios en una organización existen muchos, pero una de las herramientas que facilita todo el proceso de innovación, mejora continua o toma de decisiones definitivamente es la cadena de valor porque nos permite analizar cada procesos interno y externo que lleva a cabo la organización y a su vez examinar y estudiar cada una de las actividades que se desarrollan allí, esto quiere decir que podemos desintegrar un proceso complejo convirtiéndolo en algo simple de observación y mejora, para que este nos otorgue ventaja competitiva (diferenciación), mejora continua y de esta manera seamos capaces de competir en un mercado dominado por la competencia. Teniendo como base lo expuesto anteriormente podemos determinar que es de crucial importancia la realización de la cadena de valor de la cebolla Junca del municipio de Aquitania-Boyacá por varias razones. por ejemplo, lograr una identificación y diferenciación de la cebolla Junca del municipio con otras que se cultivan en lugares como Santander, Nariño, Valle del Cauca y Antioquia.

Logrando sistematizar un modelo de cadena de valor para la cebolla junca del municipio de Aquitania-Boyacá se podrá beneficiar a todas las personas que intervienen en el proceso ya que son personas que en su mayoría poseen hasta básica primaria, por lo cual les permite obtener conocimiento el cual les permitirá ejecutar mejoras en corto plazo y lograr un factor que no se consiga en el departamento anteriormente mencionado.

Al momento de generar las oportunidades de mejora, surgirán nuevas ideas de negocio y emprendimientos que beneficiarán a todos los involucrados en la cadena de valor, comenzando por los agricultores, quienes podrán desarrollar sus actividades con procedimientos mucho más apropiados que se adecuen a la normativa y parámetros establecidos, pero que les permita tener mayor rentabilidad; siguiendo con los clientes y consumidores finales, quienes recibirán un producto de mayor calidad, con valor agregado $\mathrm{y}$ con un precio acorde a su poder adquisitivo.

\section{REFERENCIAS}

Alcaldía Municipal de Aquitania. (2018) Nuestro municipio. [Online]. Recuperado de https://aquitaniaboyaca.micolombiadigital.gov.co/municipio/nuestro-municipio

Alonso, G. (2008). Marketing de servicios: reinterpretando la cadena de valor. Palermo Business Review, 2, 83-96. https://www.palermo.edu/economicas/pdf_economicas/business_paralela/review/marketing_servicios.pdf

Mejía, C., Soto, O. C., Gámez, H. M. y Moreno, J. P. (2015). Análisis del tamaño de empaque en la cadena de valor para minimizar costos logísticos: un caso de estudio en Colombia. Estudios Gerenciales, 31(134), 111-121. http://dx.doi. org/10.1016/j.estger.2014.06.009 
Barrera, H., Andrea, J., Espinosa, R., Janneth, A., Álvarez, S. y Pablo, J. (2019). Contaminación en el Lago de Tota, Colombia: toxicidad aguda en Daphnia magna (Cladocera: Daphniidae) e Hydra attenuata (Hydroida: Hydridae). Revista de Biología Tropical, 67(1), 11-23. http://dx.doi.org/10.15517/rbt.v67i1.33573

Bayer Crop Science. (septiembre 8, 2014). La Tierra de la Cebolla. [Online]. Disponible en https://www.cropscience.bayer.co/es-CO/Centro-de-Noticias/Noticias/2014/08/La_Tierra_de_la_Cebolla.aspx

Bejarano, J. (1998). Economía de la agricultura. Santafé de Bogotá: Tercer Mundo. De La Fuente, M. \& Muñoz, C. (2003). Ventaja competitiva:¿Actividades o recursos? Panorama socioeconómico, (26), 1-13. Disponible en http://www.panorama.utalca.cl/

DANE. (2015). La cebolla de rama o cebolla junca (Allium fistulosum), una hortaliza de gran importancia en la alimentación humana. [Online]. Recuperado de https://www.dane.gov.co/files/investigaciones/agropecuario/sipsa/Bol_Insumos_ may_2015.pdf

El Tiempo. (noviembre 26, 2016). El $64 \%$ de hogares rurales no cuentan con acceso a la tierra. eltiempo.com. [Online]. Disponible en https://www.eltiempo.com/economia/sectores/desigualdad-en-la-propiedad-de-la-tierra-en-colombia-32186

Forero, R. Á. (agosto 16, 2017). Opinión. Obtenido de Cadenas de valor y la importancia de la inserción. dinero.com. [Online]. Disponible en https://www.dinero.com/ opinion/columnistas/articulo/cadenas-de-valor-y-la-importancia-de-la-insercionraul-avila/241685

Francés, A. (2006). Estrategia y planes para la empresa: con el cuadro de mando integral. México, D.F.: Pearson Educación.

Iglesias, D. (2002). Cadenas de valor como estrategia: las cadenas de valor en el sector agroalimentario. [Documento de trabajo]. La Pampa: Estación Experimental Agropecuaria Anguil, Instituto Nacional de Tecnología Agropecuaria. Recuperado de http://www.eumed.net/ce/dhi-cadenas.pdf/www.eumed

Interempresas Media. (2019). Cebolla, allium cepa / liliaceae (alliaceae). [Online]. Obtenido de https://www.frutas-hortalizas.com/Hortalizas/Presentacion-Cebolla.html

Jiménez, M. y Molano, N. (2019). Estudio de inserción de lulo deshidratado colombiano en cadena de valor del mercado peruano. [Proyecto especialización]. Uniempresarial Fundación Universitaria Empresarial de la Camara de Comercio de Bogota, Bogotá, D.C., Colombia. Recuperado de https://bibliotecadigital.ccb. org.co/bitstream/handle/11520/23628/Molano\%20Segura\%20Andr\%C3\%A9s. pdf? sequence $=2 \&$ is Allowed $=y$

López, B. (enero 16, 2017). Origen e historia de la cebolla. [Online]. Obtenido de https://comida.uncomo.com/articulo/origen-e-historia-de-la-cebolla-44007.html

Medina, C. (agosto 15, 019). Actualidad. Obtenido de Producto Interno Bruto en el segundo semestre creció, según el Dane. wradio. [Online]. https://www.wradio. com.co/noticias/actualidad/producto-interno-bruto-en-el-segundo-semestre-crecio-segun-el-dane/20190815/nota/3940976.aspx 
Núñez, S. C. y Possetto, M. L. (2009). Estudio de la logística en el eslabón final de la cadena de valor agroindustrial en la provincia de San Luis. Revista de estudios regionales, 5, 87-124. Recuperado de http://sedici.unlp.edu.ar/bitstream/handle/10915/59974/Documento_completo.4521.pdf-des.pdf-PDFA.pdf?sequence=1

Olmedo, D. y Plazaola, N. (2018). Cadena de Valor. Estrategia Magazine, Sección administración, 1(19), 2-3. Recuperado de https://josemanuelmora.jimdo.com/ app/download/9411615069/Cadena+de+Valor+Magazine.pdf?t=1529462506

Porter, M. (1985). Competitive advantage: Creating and sustaining superior performance. New York: Free Press.

Quintero, J. y Sánchez, J. (2006). La cadena de valor: Una herramienta del pensamiento estratégico. Telos, 8(3), 377-389. Disponible en http://ojs.urbe.edu/index. php/telos/article/view/1282

Severich, G. (2012). La cadena de valor como nuevo eje de competividad frente a los desafios del mercado global. [Trabajo de Especialización]. Universidad Tecnologica de Bolivar, Cartagena de Indias. Disponible en https://repositorio.utb.edu. co/bitstream/handle/20.500.12585/2458/0062819.pdf?sequence=1

\section{BIODATA}

Jenny Mairena Herrera Rodríguez es Administradora de empresas por la universidad Pedagógica y Tecnológica de Colombia; magister en Seguridad y Salud en el Trabajo por la universidad Pedagógica y Tecnológica de Colombia. Docente investigadora de la Universidad de Boyacá (Colombia), con participación en eventos científicos nacionales e internacionales, coordinadora de semilleros de investigación, directora de trabajos de grado en modalidad proyectos de investigación, evaluadora de artículos de la revista Económicas CUC, evaluadora de trabajos en eventos científicos, organizadora de eventos científicos, integrante del grupo CIOL Uptc categoría A. Áreas de interés: Administración, desempeño laboral, responsabilidad social, mercadeo, innovación, SST. https://orcid.org/0000-0002-9809-5604

Wilmar Nicolas Chacón Casallas es estudiante de terminación académica de la Universidad de Boyacá (Colombia) y estudiante del semillero JET-BM. Con intereses en la investigación, Finanzas, recurso humano, contabilidad, innovación, estrategia, economía y gerencia. Desarrollando proyecto titulado modelo de cadena de valor de la cebolla larga de Aquitania Boyacá. https://orcid.org/0000-0002-1249-0874

Julián Andrés Aragón García es estudiante de terminación académica de la Universidad de Boyacá (Colombia), perteneciente al grupo semillero JET-BM intereses de investigación: Finanzas, innovación, cadena de valor, organizacional, economía y gerencia. https://orcid.org/0000-0002-3095-6826 